\title{
Cooperative interception strategy for multiple inferior missiles against one highly maneuvering
}

\author{
target \\ Wenshan $\mathrm{Su}^{\mathrm{a}}$, Hyo-Sang Shin ${ }^{\mathrm{b} \dagger}$, Lei Chen ${ }^{\mathrm{a} \ddagger}$, Antonios Tsourdos ${ }^{\mathrm{b} \S}$ \\ ${ }^{a}$ National University of Defense Technology, Changsha, People's Republic of China \\ ${ }^{\mathrm{b}}$ Cranfield University, Cranfield, United Kingdom
}

\begin{abstract}
This paper proposes a novel cooperative guidance strategy, which aims to intercept one highly maneuvering target with multiple inferior missiles. In the scenario of interest, both the missiles and target are assumed to have bounded maneuverability, and the guidance goal is to make the joint reachable set of interceptors cooperatively cover the target maneuvering range. Under this guidance scheme, a preprogrammed covering strategy and an adaptive covering strategy are designed for the missile teams without and with communication capability respectively. The former attempts to specify different subsets of the target maneuvering range to different missiles as the expected reachable sets, while the latter aims to coordinate the expected reachable sets of different missiles dynamically according to the changing engagement situation. Considering the disadvantage of inferior maneuverability, the inherent limitations of the proposed guidance scheme are discussed. Numerical simulations with different target maneuvering modes demonstrate the prominent performance improvements of cooperative strategy over the traditional guidance laws.
\end{abstract}

Keywords: Cooperative interception; Coverage-based guidance law; Virtual aiming point

\section{Introduction}

Traditionally, the guidance goal mainly focuses on how to guide single missile to the specified point with limited measuring information, and a series of guidance laws have been developed to continuously improve the guidance performance. One important branch of this guidance theme consists of the

\footnotetext{
* Ph. D. Student, College of Aerospace Science and Engineering; suwenshan12@nudt. edu.cn.

$\dagger$ Senior Lecturer, School of Aerospace, Transport and Manufacturing, h.shin@cranfield.ac.uk

$\$$ Professor, College of Aerospace Science and Engineering; chenl@nudt.edu.cn

$\S$ Professor, School of Aerospace, Transport and Manufacturing, a.tsourdos@cranfield.ac.uk
} 
proportional navigation guidance law (PNG) and its various extensional forms, such as the true proportional navigation law (TPN) ${ }^{[1]}$, pure proportional navigation law (PPN) ${ }^{[2]}$, augmented proportional guidance law $(\mathrm{APN})^{[3]}$, and so $\mathrm{on}^{[4]}$. The guidance laws, which are based on the optimal control ${ }^{[5]}$, differential games ${ }^{[6][7]}$, and other modern control theories ${ }^{[8][9]}$, are also one important branch. There is no doubt that a great progress has been made by these guidance laws when attacking the traditional targets, whose maneuverability is significantly inferior to that of the missiles. However, all of them will face significant challenges with the dramatic advancement of target countermeasures, such as the defense capability, camouflage ability, maneuverability and so on ${ }^{[10]}$. For example, the appearance of the hypersonic flight vehicle will degrade the performance of many existing intercepting missiles, because of its high speed and substantial maneuverability potential. One promising solution is the cooperative guidance law, which can offset the deficient counter-countermeasure of single missile by coordinating the action of different missiles.

One typical application of cooperative guidance laws is the salvo attack. Generally, the salvo guidance laws can be classified into two categories in terms of the methods of achieving simultaneous impact: 1) preprogramming the impact time before launch; 2) synchronizing the impact time during the flight. The pioneering work of the first category is done by Jeon et al ${ }^{[11]}$, who succeeded to make the flight time approach the programmed impact time by superimposing one feedback of impact time error on PNG. Further, Lee et al. ${ }^{[12]}$ and Harl et al. ${ }^{[13]}$ derived this kinds of guidance laws via optimal control and sliding mode control, respectively. However, one evident drawback of the first category is that it is difficult to specify the preprogrammed time properly for the moving target, especially the uncertain maneuvering target, and then the second category is proposed. To synchronize the impact time dynamically, the leaderfollower strategy ${ }^{[14]}$ and the centralized coordination algorithms ${ }^{[15]}$ are proposed. Furthermore, to improve the adaptability and extensibility of the guidance system, Zhao et al. ${ }^{[16]}$ presented a distributed coordination algorithm based on the impact-time-control guidance law ${ }^{[1]}$.

Cooperative interception of the confusing target, which may result from the decoys or the uncertain target maneuver, is another important research topic. Unlike the conventional work, the predicted target position at the interception is described by a region with certain probability density function (PDF) rather than a certain point, and the guidance goal is formulated as maximizing the interception probability instead of nullifying the miss distance ${ }^{[17][18]}$. Zhai et al. ${ }^{[19]}$ considered the problem of multiple interceptors against the target with decoys, and proposed a coverage-based interception algorithm to 
maximize the joint interception probability. Vitaly et al. ${ }^{[20]}$ presented a cooperative strategy between the leading missile and trailing missiles, and substantially improved the interception performance of the trailing missiles by staggering missile launch and sharing target information. Similarly, to decrease the handover error, Wang et al. ${ }^{[21]}$ developed one cooperative mid-course guidance scheme, i.e., making the missiles in the middle phase use the target information collected by the missiles in the terminal phase.

However, all the guidance laws ${ }^{[19-22]}$ are designed by assuming the superiority of interceptor's maneuverability, which can be hardly guaranteed when intercepting a highly maneuvering target, such as the hypersonic flight vehicle. Therefore, this paper will develop a cooperative guidance strategy for multiple inferior missiles to intercept a highly maneuvering target. Instead of assuming the target acceleration is known, which is almost impossible for the uncertain maneuvering target, we only regard the upper boundary of the target acceleration as the prior information. Under this assumption, the guidance goal is transformed into maximally covering the bounded target maneuvering range by coordinating the reachable sets of different missiles, and the corresponding cooperative guidance strategies for the missile team without and with communication capability are designed respectively.

The remainder of this paper is organized as follows. Section II is the problem description, where the linearized kinematics between interceptors and target is built and the guidance goal of cooperative covering target maneuvering range is introduced. In section III, considering the missile team without and with communication ability, two different cooperative guidance strategies are designed and analyzed. Section IV illustrates the performances of the proposed guidance strategies through the simulations with different target maneuvering modes.

\section{Problem Description}

\section{A. Linearized Kinematics}

A schematic view of the planar endgame geometry between multiple intercepting missiles and target is shown in Fig. 1, where $O_{I} X_{I} Y_{I}$ is a Cartesian inertial reference frame. The notations $M$ and $T$ represent the intercepting missile and target, respectively. The velocity and normal acceleration are denoted by $v$ and $a$. The parameters with subscripts $i, j$, or $T$ correspond to the $i^{\text {th }}$ and $j^{\text {th }}$ missile and the target. $r_{i}$ is the range between the $i^{\text {th }}$ missile and target, $q_{i}$ is the angle between LOS and the $X_{I}$ axis, $\theta_{i}$ is the flight path angle. The initial LOS of homing phase is denoted by $\operatorname{LOS}_{i 0}$, 
which will be used for linearization. $q_{i 0}$ means the angle of $\operatorname{LOS}_{i 0}$ with respect to $X_{I}$. The engagement kinematics between the $i^{\text {th }}$ missile and target can be expressed as

$$
\left\{\begin{array}{l}
\dot{r}_{i}=v_{T} \cos \left(\theta_{T}-q_{i}\right)-v_{i} \cos \left(\theta_{i}-q_{i}\right) \\
\dot{q}_{i}=v_{T} \sin \left(\theta_{T}-q_{i}\right) / r_{i}-v_{i} \sin \left(\theta_{i}-q_{i}\right) / r_{i}
\end{array}\right.
$$

Assuming the control dynamics of both target and interceptors are ideal, we have

$$
\left\{\begin{array}{l}
\dot{\theta}_{T}=a_{T} / v_{T} \\
\dot{\theta}_{i}=a_{M, i} / v_{i}
\end{array}\right.
$$

Let $y_{i}$ be the component of $r_{i}$ normal to $\operatorname{LOS}_{i 0}$, as shown in Fig. 1, and the second derivative of $y_{i}$ is

$$
\ddot{y}_{i}=-a_{T} \cos \left(\theta_{T}-q_{i 0}\right)-a_{M, i} \cos \left(\theta_{i}-q_{i 0}\right)
$$

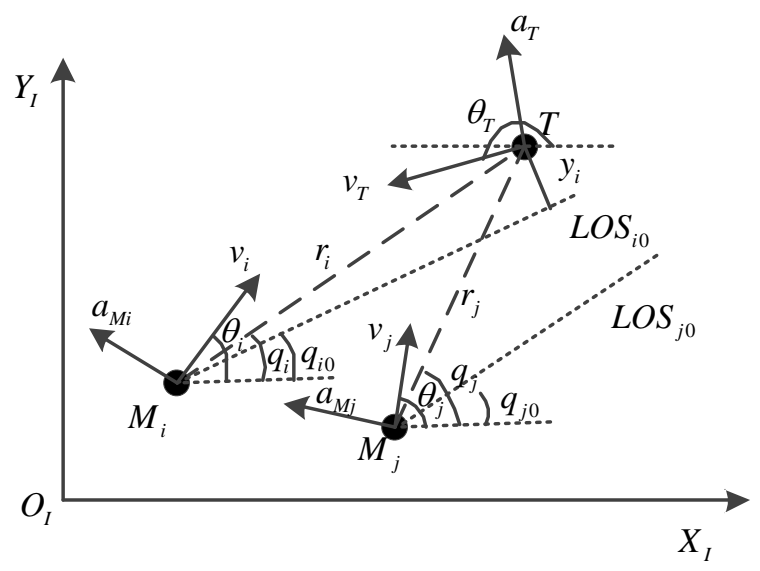

Fig. 1 Planar engagement geometry

During the endgame, usually the deviations of $\theta_{T}$ and $\theta_{i}$ from the initial collision triangle are small

[4], so it is acceptable to regard them as constant. Therefore, we can rewrite the above equation as

$$
\ddot{y}_{i}=L_{T} a_{T}-L_{M i} a_{M i}
$$

where $L_{T}$ and $L_{M i}$ are the constant coefficients

$$
\left\{\begin{array}{l}
L_{T}=-\cos \left(\theta_{T}-q_{i 0}\right) \\
L_{M i}=\cos \left(\theta_{i}-q_{i 0}\right)
\end{array}\right.
$$

In particular, for the head-on scenario, the values of $L_{T}$ and $L_{M i}$ are

$$
L_{T}=1, L_{M i}=1
$$

Known from Ref. [4], for the maneuvering target with ideal control dynamics, the zero effort miss distance $Z E M_{i}$ of the $i^{\text {th }}$ interceptor could be defined as 


$$
Z E M_{i}(t)=y_{i}(t)+\dot{y}_{i}(t) t_{g o, i}+\int_{t}^{t_{f, i}} a_{T} t_{g o, i} d t
$$

where $t_{g o, i}$ is the time to go, and could be approximated by $\left|r_{i} / \dot{r}_{i}\right|$ under the constant-speed assumption, $t_{f, i}$ denotes the total flight time of the terminal phase.

For convenience of the flowing analysis, we also define

$$
Z E M_{i}^{*}(t)=y_{i}(t)+\dot{y}_{i}(t) t_{g o, i}
$$

which represents the predicted miss distance without considering the target evading acceleration and missile control effort.

\section{B. Guidance Goal}

Traditionally, the guidance law aims to eliminate the nonzero $Z E M$ and enables the interceptor to reach the predicted collision point, which essentially needs to keep the target in the missile reachable set. Inspired by this, we can also describe the cooperative guidance goal as maintaining the target evasion region in the joint reachable set of the missile team. Fig. 2 illustrates this idea in the coordinate frame $O X Y$, whose axes are parallel to that of the initial LOS coordinate system and origin is centered at the target.

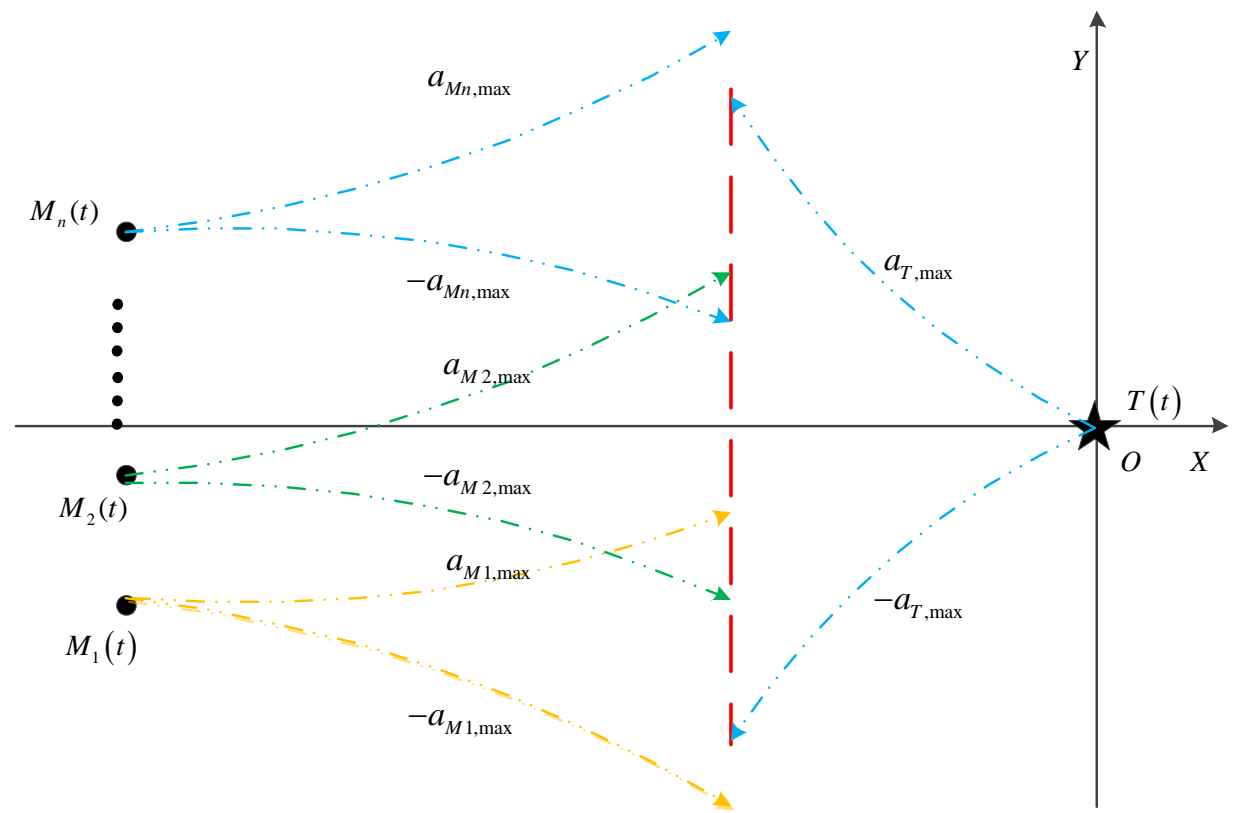

Fig. 2 Schematic of the cooperative strategy

From Ref. [22], the necessary condition of zero miss distance for missile $M_{i}$ is the whole distributions of ZEM must lie inside its maximum position divert envelope, that is 


$$
\left|Z E M_{i}(t)\right| \leq a_{M i, \max } t_{g o, i}{ }^{2} / 2
$$

where $a_{M i, \max }$ is the maximum maneuverability of missile $M_{i}$.

Combining the above equation with Eq. (7), we can derive the admissible target maneuvering acceleration for the missile $M_{i}$.

$$
\left\{\begin{array}{l}
R_{i}(t) \triangleq\left[a_{i t, \text { low }}(t), a_{i t, u p}(t)\right] \\
a_{i t, \text { up }}(t)=a_{M i, \max }-2 Z E M_{i}^{*}(t) / t_{g o, i}{ }^{2} \\
a_{i t, \text { low }}(t)=-a_{M i, \text { max }}-2 Z E M_{i}^{*}(t) / t_{g o, i}{ }^{2}
\end{array}\right.
$$

which represents the reachable set of missile $M_{i}$ in terms of the allowable target acceleration when $M_{i}$ makes full use of its maneuverability.

Define the joint reachable set of multiple missiles as

$$
R_{M}(t)=\bigcup_{i=1}^{n} R_{i}(t)
$$

Note that the target maneuvering range is

$$
E_{T}(t)=\left[-a_{T, \max }, a_{T, \max }\right]
$$

Hence, the necessary condition for the successful interception of the missile team is there always exists at least one feasible control input

$$
\left|a_{M i}(t)\right| \leq a_{M i, \max }, \quad \exists i \in\{1,2,3 \ldots n\}, t_{0} \leq t \leq t_{f}
$$

can guarantee

$$
\left|Z E M_{i}(t)\right| \leq a_{M i, \max } t_{g o, i}{ }^{2} / 2, \quad \forall\left|a_{T}(t)\right| \leq a_{T, \max }, t_{0} \leq t \leq t_{f, i}
$$

where $t_{0}$ denote the initial moment of homing phase. As shown in Fig. 2, the possible target position at the interception is within the joint divert envelope of missiles. Essentially, this above condition is equivalent to the following constraint.

$$
E_{T}(t) \subseteq R_{M}(t)
$$

It is evident the target would be intercepted by the missile group if the above expression remains valid during the total homing phase. Therefore, we will regard the above constraint as the cooperative guidance goal. 


\section{Cooperative Guidance Strategy}

For fulfilling the cooperative guidance goal above, the key is to design one strategy to coordinate the reachable sets of different missiles. In this section, firstly we will propose a preprogrammed strategy of covering the target maneuvering range, which can be applied to the missile team without communication capability. While for the missile group with communication ability, a covering strategy that can adapt to the changing engagement situation is developed.

\section{A. Preprogrammed Covering Strategy}

For the missile group without communication capability, the cooperative strategy needs to be preprogrammed before launch and cannot be changed again. Assuming the missile-team consists of $n$ missiles, and all the missiles have the same maneuverability $a_{M, \max }$. For convenience of the following analysis, the missiles are numbered as $M_{1}, M_{2}, \ldots$ and $M_{n}$ according to descending order of their $\operatorname{ZEM}_{i}^{*}\left(t_{0}\right)$.

According to Eq. (10), the span of the reachable set of single missile is $2 a_{M, \max }$, while the maneuvering range of the target is $2 a_{T, \max }$ according to Eq. (12). Therefore, to cover the whole target maneuvering range, the number of missiles should meet

$$
n \geq\left\lceil a_{T, \text { max }} / a_{M, \text { max }}\right\rceil
$$

where the notation $\lceil\cdot\rceil$ represents the function that returns a number rounded upwards to the nearest integer. Correspondingly, we can equally divide the target maneuvering range into $n$ parts, and assign them to different missiles, as shown in Fig. 3.

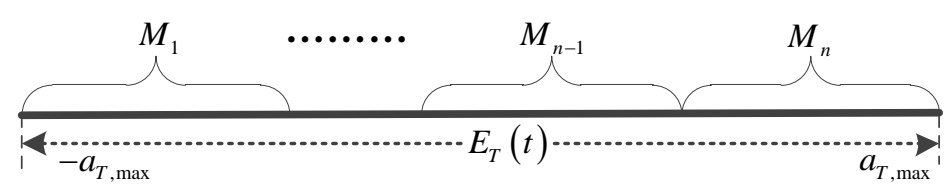

Fig. 3 Ideal covering strategy

Mathematically, the assigned covering area $C_{i}$ of each missile is defined as

$$
\left\{\begin{array}{l}
C_{i}=\left[C_{i, \text { low }}, C_{i, \text { up }}\right] \\
C_{i, \text { low }}=-a_{T, \max }+2(i-1) a_{T, \max } / n, i=1,2 \ldots . n \\
C_{i, \text { low }}=C_{i, \text { low }}+2 a_{T, \max } / n
\end{array}\right.
$$


To guarantee $\left[C_{i, l o w}, C_{i, u p}\right]$ is within the reachable set of $M_{i}$ at the beginning, the middle guidance should ensure the initial state of $M_{i}$ to meet the following constraints according to Eq. (10).

$$
\left\{\begin{array}{l}
a_{i t, l o w}\left(t_{0}\right) \leq C_{i, l o w} \\
a_{i t, u p}\left(t_{0}\right) \geq C_{i, u p}
\end{array}\right.
$$

That is

$$
\frac{-a_{M i, \max }-C_{i, u p}}{2} t_{f, i}{ }^{2} \leq Z E M_{i}^{*}\left(t_{0}\right) \leq \frac{a_{M i, \max }-C_{i, u p}}{2} t_{f, i}{ }^{2}
$$

which implies the requirement on the handover errors of middle guidance. If the handover errors of all the missiles satisfy the above constraint, then we have

$$
E_{T}\left(t_{0}\right) \subseteq R_{M}\left(t_{0}\right)
$$

Due to lack of mutual communication, we cannot readjust the assigned covering areas according to the change of engagement situation. Instead, what we can do is to make each missile full use its maneuverability to cover the assigned area, and to maximize its interception probability. If this successes, $E_{T}\left(t_{0}\right) \subseteq R_{M}\left(t_{0}\right)$ can extend to be $E_{T}(t) \subseteq R_{M}(t), t_{0} \leq t \leq t_{f}$ and the interception is guaranteed. With this motivation, the preprogrammed covering strategy is developed as follows.

Firstly, define the interception probability of each missile as

$$
p_{i}(t)=\frac{S\left(R_{i}(t) \cap E_{T}(t)\right)}{S\left(E_{T}(t)\right)}
$$

where the function $S(\bullet)$ represents the span of the set. From this definition, the maximum value of $p_{i}$ is

$$
\max \left(p_{i}\right)=\min \{1, \mu\}, \mu=a_{M, \max } / a_{T, \max }
$$

where the maximum interception probability can reach 1 if the maneuverability of missile is superior to that of the target, otherwise it is $\mu$. Herein we only consider the case that missile maneuverability is inferior to that of the target. In this case, considering the assigned covering area, it is intuitive that the expected reachable set $R_{i}^{*}$ of each missile should completely lie in the target maneuvering range, as shown in Fig. 4. 


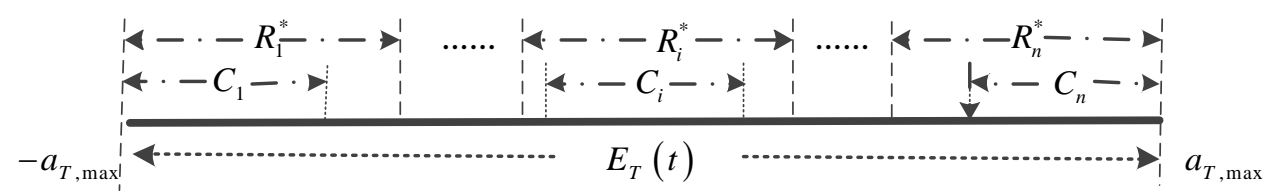

Fig. 4 Expected reachable sets

Note that the expected reachable sets corresponding to $M_{1}$ and $M_{n}$ are unique, while there are many choices for the other missiles, such as $M_{i}$ in Fig. 4. To avoid the non-uniqueness, we design the expected reachable set of each missile under the priority of following principles: 1) it contains the assigned covering subsection; 2) it is in the target maneuvering range; 3 ) the distance between the centers of $R_{i}^{*}$ and $C_{i}$ should be as small as possible. Mathematically, we can define the expected reachable set as

$$
\left\{\begin{array}{l}
R_{i}^{*}=\left[a_{i t, l o w}^{*}, a_{i t, u p}^{*}\right] \\
a_{i t, l o w}^{*}=\max \left\{\frac{C_{i, l o w}+C_{i, u p}}{2}-a_{M, \max },-a_{M, \max }\right\} \& a_{i t, u p}^{*}=a_{i t, l o w}^{*}+2 a_{M, \max }, \text { if } a_{i t, u p}^{*} \leq a_{T, \max } \\
a_{i t, u p}^{*}=\min \left\{\frac{C_{i, l o w}+C_{i, u p}}{2}+a_{M, \max }, a_{M, \max }\right\} \& a_{i t, l o w}^{*}=a_{i t, u p}^{*}-2 a_{M, \max }, \text { if } \quad a_{i t, l o w}^{*} \geq-a_{T, \max }
\end{array}\right.
$$

According to Eq. (10), the expected $Z_{E M_{i}^{*}}^{*}(t)$ corresponds to $R_{i}^{*}$ is

$$
Z E M_{i, \exp }^{*}=-\frac{a_{i t, u p}^{*}+a_{i t, l o w}^{*}}{2} t_{g o . i}^{2}
$$

Motivated by differential game guidance law ${ }^{[23-25]}$, we design the following guidance law.

$$
a_{M i}(t)=a_{M, \max } \operatorname{sign}\left(Z E M_{i}^{*}-Z E M_{i, \exp }^{*}\right)
$$

where $\operatorname{sign}(\bullet)$ represents the sign function. Under the control of the above guidance law, the missile will make full use of its maneuverability to force its current reachable set to approach $R_{i}^{*}$. Also, we can see each missile can run the above guidance law independently because it does not need other missiles' information. As a result, the programmed covering strategy is applicable for the missile team without communication capability.

\section{B. Adaptive covering strategy}

Supposing the missiles can communicate with their two adjacent missiles, that is the missile $M_{i}(1<i<n)$ has access to missiles $M_{i-1}$ and $M_{i+1}$, while $M_{1}$ and $M_{n}$ can communicate with $M_{2}$ and $M_{n-1}$ respectively. For the missile team with this kind of communication capability, instead 
of using the rigid preprogrammed covering strategy, we will design a new algorithm to dynamically adjust the covering strategy according to the current engagement situation, which will greatly improve adjustability and flexibly.

First, the requirement on the handover errors of middle guidance can be relaxed to be

$$
\exists\left|\operatorname{ZEM}_{i}\left(t_{0}\right)\right| \leq a_{M i, \max } t_{f}^{2} / 2, i=1,2,3 \quad \forall\left|a_{t}(t)\right| \leq a_{T, \max }
$$

which can guarantee $E_{T}\left(t_{0}\right) \subseteq R_{M}\left(t_{0}\right)$. Evidently, the requirement shown in Eq. (19) is a special case of the Eq. (26).

As for the expected reachable sets of different missiles, to maximize the joint interception probability of the missile team and the interception probability of each single missile, they will be designed under the following algorithm. Firstly, the expected reachable sets of all the missiles are initialized by their initial reachable sets, i.e., $R_{i}^{*}\left(t_{0}\right)=R_{i}\left(t_{0}\right)$.Then they are adjusted according to the current reachable sets of their own and their adjacent missiles. Concretely, if the current reachable sets partly lie in the target maneuvering range, the expected reachable sets are set to be

$$
\begin{cases}R_{i}^{*}\left(t_{k}\right)=\left[a_{T, \text { max }}, a_{T, \text { max }}-2 a_{M, \text { max }}\right], & \text { if } a_{T, \text { max }} \in R_{i} \\ R_{i}^{*}\left(t_{k}\right)=\left[-a_{T, \text { max }},-a_{T, \text { max }}+2 a_{M, \text { max }}\right], & \text { if }-a_{T, \text { max }} \in R_{i}\end{cases}
$$

where $t_{k}$ means the current moment. While for the missiles whose current reachable sets completely lie in the target maneuvering range, their expected reachable sets $R_{i}^{*}=\left[a_{i t, l o w}^{*}, a_{i t, u p}^{*}\right]$ are defined according to the reachable sets of their adjacent missiles, as shown in Fig. 5, 6 and 7. In Fig. 5, part of $R_{i-1}$ overlaps $R_{i}$, while there is a gap between $R_{i}$ and $R_{i+1}$. In this case, minimizing the gap and keeping intersection set between $R_{i-1}$ and $R_{i}$ nonempty will be helpful to enlarge the joint set of $R_{i-1}$, $R_{i}$, and $R_{i+1}$.Consequently, considering the boundaries of target maneuvering range the expected reachable set $R_{i}^{*}$ is designed as shown in Eq. (28). Fig. 6 illustrates the similar case, and Eq.(29) provides the corresponding expected reachable set. In Fig. 7 (a), there are two gaps among $R_{i-1}, R_{i}$ and $R_{i+1}$, while there are two overlaps in Fig. 7(b). It is obvious no matter whether $R_{i}$ moves to $R_{i-1}$ or $R_{i+1}$, the size of their joint reachable set will not change, therefore the expected reachable set remains unchanged, as shown in Eq. (30).

case 1: $\left\{\begin{array}{l}a_{i t, \text { up }}^{*}\left(t_{k}\right)=\min \left(a_{i-1 t, u p}\left(t_{k}\right)+2 a_{M, \text { max }}, a_{T, \text { max }}\right) \\ a_{i, \text { low }}^{*}\left(t_{k}\right)=a_{i t, \text { up }}^{*}\left(t_{k}\right)-2 a_{M, \text { max }}\end{array}, R_{i-1} \cap R_{i} \neq \varnothing \& R_{i+1} \cap R_{i}=\varnothing \& i>1\right.$ 
case 2: $\left\{\begin{array}{l}a_{i t \text { low }}^{*}\left(t_{k}\right)=\max \left(a_{i+1 t, \text { low }}\left(t_{k}\right)-2 a_{M, \text { max }},-a_{T, \text { max }}\right) \\ a_{i t, \text {, }}^{*}\left(t_{k}\right)=a_{i t, \text { low }}^{*}\left(t_{k}\right)+2 a_{M, \text { max }}\end{array}, R_{i-1} \cap R_{i}=\varnothing \& R_{i+1} \cap R_{i} \neq \varnothing \& i<n\right.$

$$
\text { case 3: } R_{i}^{*}\left(t_{k}\right)=R_{i}^{*}\left(t_{k-1}\right) \quad \text {,other conditions }
$$

where $a_{i-1 t, u p}\left(t_{k}\right)$ and $a_{i+1 t, l o w}\left(t_{k}\right)$ represents the boundaries of the current reachable sets of missiles $M_{i-1}$ and $\quad M_{i+1}$, and they can be calculated according to Eq. (10), $t_{k-1}$ denotes the last moment and will be $t_{0}$ if $t_{k}=t_{0}$.

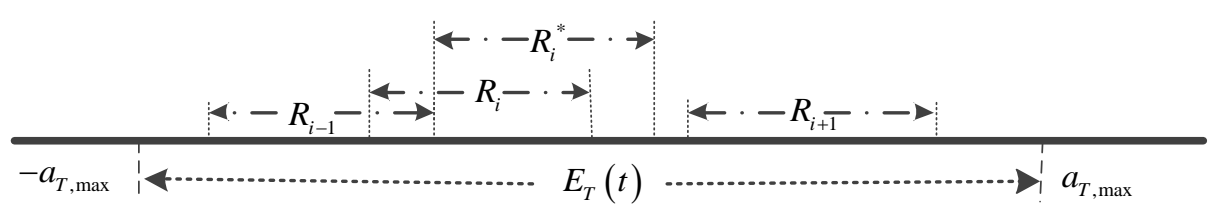

Fig. 5 Expected reachable set under case 1

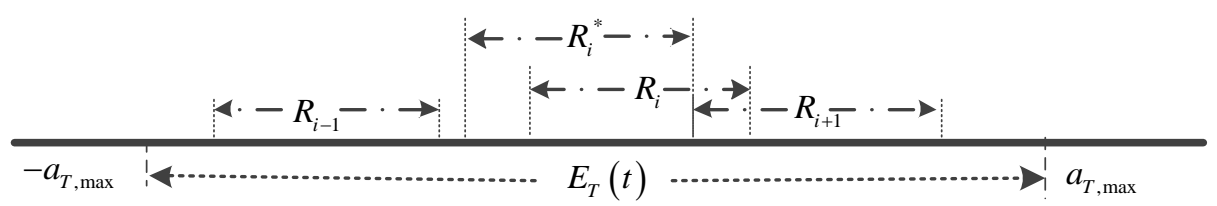

Fig. 6 Expected reachable set under case 2

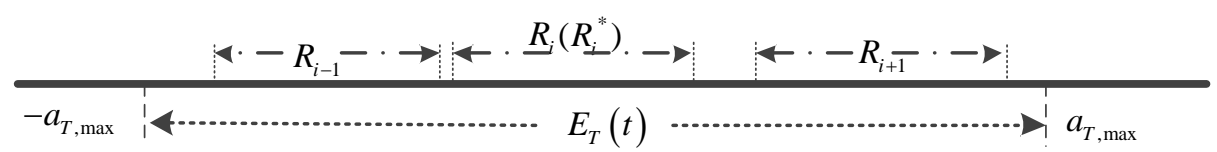

case $3(a)$

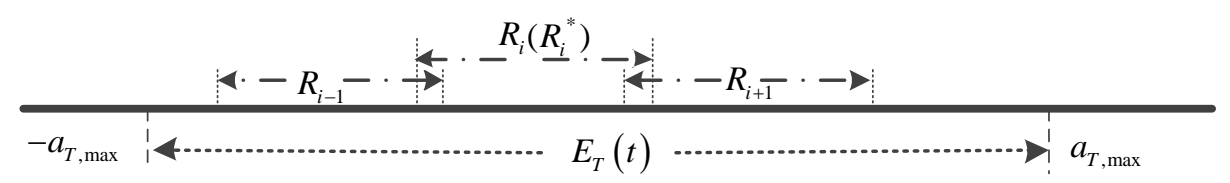

case $3(b)$

Fig. 7 Expected reachable set under case 3

In addition to the above cases, it is also possible that the current reachable sets are completely outside the target maneuvering range, which implies the interception probabilities are zero for these missiles. Hence, in this case, their interception probabilities will remain zero no matter how to design their expected reachable sets. In practice, we can define them as $R_{i}^{*}\left(t_{k}\right)=R_{i}^{*}\left(t_{k-1}\right)$.

Similar to the preprogrammed covering strategy, we can deduce the expected $Z E M_{i, \text { exp }}^{*}\left(t_{k}\right)$ according to Eq. (24) and adopt the guidance law Eq. (25) to approach the expected reachable sets.

\section{Inherent limitations of the cooperative strategy}


As mentioned in section II. B the interception will be guaranteed if $E_{T}(t) \subseteq R_{M}(t)$ remains valid during the whole homing phase. Unfortunately, it is impossible for the missile team with inferior maneuverability $a_{M, \max }$. For example, one missile team consists of $n$ missiles with maneuverability inferior to that of the target. Suppose $E_{T}(t) \subseteq R_{M}(t)$ is valid when $t=t_{1}$. Without loss of generally, according to Eqs. (13) and (14), we assume

$$
\begin{cases}\cdots & \\ R_{i}\left(t_{1}\right)=\left[a_{i t, l o w}\left(t_{1}\right), a_{i t, u p}\left(t_{1}\right)\right] & , a_{i t, \text { low }}\left(t_{1}\right) \leq-a_{T, \max }, 1 \leq i<n \\ \cdots & \\ R_{j}\left(t_{1}\right)=\left[a_{j t, \text { low }}\left(t_{1}\right), a_{j t, u p}\left(t_{1}\right)\right] & , a_{j t, \text { low }}\left(t_{1}\right) \geq a_{T, \max }, 1 \leq j \leq n \\ \cdots & \end{cases}
$$

Let the target evade at the acceleration.

$$
a_{t}(t)=a_{T, \max }, t_{1} \leq t \leq t_{f}
$$

Then the missiles with $a_{T, \max } \notin R_{j}\left(t_{1}\right)$ or the missiles with $a_{T, \max } \in R_{i}\left(t_{1}\right)$ but using inappropriate guidance laws, their final miss distances will not be zero. Hence, there must exist one moment $t_{2}=t_{f}-\delta t$ meeting the following inequality for these missiles.

$$
\left|\frac{Z E M_{i}^{*}(t)}{t_{g o}{ }^{2}}\right|>>a_{M, \max }, t_{2}<t<t_{f}
$$

Combining with Eq. (10), the above inequality implies that their corresponding reachable sets meet

$$
R_{i}(t) \cap E_{T}=\varnothing, t_{2}<t<t_{f}, \text { if } \operatorname{ZEM}_{j}\left(t_{f}\right) \neq 0
$$

which implies $-a_{T, \max }$ is not in their reachable sets.

While for the missiles with $a_{T, \max } \in R_{j}\left(t_{1}\right)$ they will have zero miss distance if they are under the control effort of appropriate guidance laws. However, it will be impossible for them to guarantee $-a_{T, \max } \in R_{j}(t), t_{2}<t<t_{f} \quad$ simultaneously because $a_{M, \max } \leq a_{T, \max }$. That is

$$
-a_{T, \max } \notin R_{j}(t), t_{2}<t<t_{f}, \text { if } Z E M_{j}\left(t_{f}\right)=0
$$

Combining this with Eq.(34), we have $-a_{T, \max } \notin R_{M}(t), t_{2}<t<t_{f}$, and can make the conclusion that there must exist one moment $t_{2}$ to make $E_{T}(t) \subseteq R_{M}(t), t_{2}<t<t_{f}$ invalid if the maneuverability of 
the missile team satisfies $a_{M, \max } \leq a_{T, \max }$, no matter what cooperative strategy it adopts.

However, when it comes to certain target maneuvering modes, failure of keeping $E_{T}(t) \subseteq R_{M}(t)$ does not definitely result in the failure of interception. If the target executes constant maneuver, it can still be intercepted by the missile group adopting the proposed cooperative strategies as long as $E_{T}\left(t_{0}\right) \subseteq R_{M}\left(t_{0}\right)$, although the target's maneuverability is superior to that of missiles. In addition, the missile group also can achieve the successful interception if the target's actual maneuver lies in certain range. For example, for the preprogrammed covering strategy, the interception can be guaranteed as long as $a_{t}(t)$ remains in the assigned covering areas of any missile, i.e., $\forall i \in\{1, \ldots n\}: a_{t}(t) \in C_{i}$. While for the adaptive covering strategy this is also valid when $\forall i \in\{1, \ldots n\}: a_{t}(t) \in R_{i}\left(t_{0}\right)$. As for the other conditions, such as the bang-bang maneuver, the interception may not always be guaranteed by the proposed cooperative strategy but the interception probability will be greatly increased compared with other traditional guidance laws. All the above conclusions will be proved through the flowing simulations.

\section{Simulations and Analysis}

In this section, the guidance performance of the cooperative strategies will be verified through a series of numerical simulations. For all the following scenarios, the missile team is assumed to consist of three missiles $M_{1}, M_{2}$, and $M_{3}$. All the missiles have the same initial positions $[0,0] \mathrm{km}$, the velocities corresponding to missiles $M_{1}, M_{2}$, and $M_{3}$ are $[2,-0.04] \mathrm{km} / \mathrm{s},[2,0] \mathrm{km} / \mathrm{s}$, and $[2,0.04] \mathrm{km} / \mathrm{s}$, while the initial position and velocity of the target are $(50,0) \mathrm{km}$ and $(-3,0) \mathrm{km} / \mathrm{s}$, respectively. The maximum accelerations corresponding to the target and missiles are $a_{T, \max }=4 g$ and $a_{M, \max }=3.2 \mathrm{~g}$, i.e., $\mu=0.8$. According to these initial states, we can calculate the initial $Z E M$ s of $M_{1}, M_{2}$, and $M_{3}$, i.e., $400 \mathrm{~m}, 0 \mathrm{~m}$ and $-400 \mathrm{~m}$ respectively, which ensures the precondition shown Eq. (20).

Firstly, consider the scenario where the target executes constant maneuver

$$
a_{t}=u a_{T, \max } \cdot U(t-\tau),|u| \leq 1
$$

where $U(t-\tau)$ represents a unit step function starting at $\tau$, and here $\tau$ is set to be 0 meaning the maneuver starts at the beginning, the sign of $u$ means the maneuvering direction. 


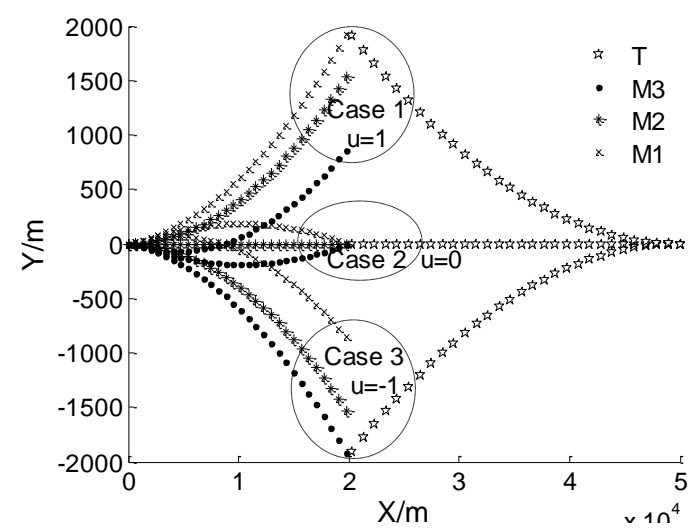

Fig. 8 Flight trajectories of missiles and constant maneuvering target

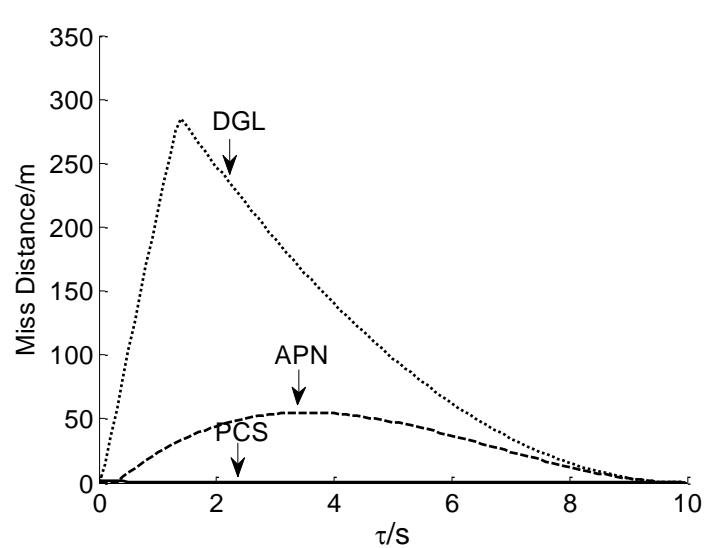

Fig. 9 Miss distance against the random step maneuver: $\|u\|=1$

Let the missile team adopt the preprogrammed covering strategy, Fig. 8 shows the fight trajectories of missiles and targets under the cases $u=1, u=0$, and $u=-1$.we can see targets in cases $u=1$ and $u=-1$ are intercepted by the missile $M_{3}$ and $M_{1}$ respectively, while the target with $u=0$ can be intercepted by all the three missiles. For the results that the targets in cases 1, 2, and 3 can be intercepted by missiles $M_{3}, M_{2}$ and $M_{1}$, respectively, it is simply because the target maneuvering accelerations are in the assigned covering areas of the corresponding missiles according to Eq. (17).

$$
\left\{\begin{array}{l}
C_{1}=\left[-a_{T, \max },-a_{T, \max } / 3\right] \\
C_{2}=\left[-a_{T, \max } / 3, a_{T, \max } / 3\right] \\
C_{3}=\left[-a_{T, \max } / 3, a_{T, \text { max }}\right]
\end{array}\right.
$$

While the reason why missile $M_{1}$ and $M_{3}$ also can intercept the target of case 2 is that their expected reachable sets contains $u=0$ according to Eq . (23).

$$
\left\{\begin{array}{l}
R_{1}^{*}=\left[-a_{T, \text { max }}, 3 a_{T, \text { max }} / 5\right], \\
R_{3}^{*}=\left[-3 a_{T, \text { max }} / 5, a_{T, \text { max }}\right],
\end{array}\right.
$$

Meantime, we can find $u=0$ is also in their initial reachable sets according to Eq. (9). Hence, under the guidance law Eq. (25), missiles $M_{3}$ and $M_{1}$ can always keep the target in their reachable sets during the homing phase, and intercept it finally.

Taking into account the random step maneuver ${ }^{[4]}$ whose starting time is uniformly distributed over the whole flight time, i.e., $\|u\|=$ const and $0 \leq \tau \leq t_{f}$ for Eq. (36). It is easy to find the constant maneuver is one special case of random step maneuver. Define the minimum miss distance of three missiles as the miss distance of the missile team. Fig. 9 shows the miss distances of missile team under different starting times. The curves labeled by PCS, APN and DGL correspond to the results of the preprogrammed 
covering strategy, the augmented proportional navigation guidance ${ }^{[4]}$, and the differential game guidance law ${ }^{[25]}$.

$$
\begin{aligned}
& A P N: a_{i}(t)=N \frac{Z E M_{i}^{*}(t)}{t_{g o, i}{ }^{2}}+0.5 N \hat{a}_{t}(t) \\
& D G L: a_{i}(t)=\operatorname{sign}\left(Z E M_{i}(t)\right), Z E M_{i}(t)=y_{i}(t)+\dot{y}_{i}(t) t_{g o, i}+\hat{a}_{t}(t) t_{g o, i}{ }^{2} / 2
\end{aligned}
$$

where $\hat{a}_{t}(t)$ denotes the estimated value of target acceleration. For simplicity, we set $\hat{a}_{t}(t)$ to be the true value of target acceleration, i.e., $\hat{a}_{t}(t)=a_{t}(t)$. The guidance gain $N$ of APN is set to be 3 .

From Fig. 9, we can see the miss distances of PCS remain at small values for different starting times, while the miss distances of APN and DGL are significantly affected by the starting time. This difference mainly results from that APN, DGL and PCS have different predictions about the target future acceleration. For APN and DGL, the guidance laws of all the missiles are designed with the same prediction that the target will keep maneuvering at its current acceleration for the time to go. While in PCS different expected reachable sets are designed for different missiles to intercept the targets within different maneuvering ranges. Concretely, according to Eq. (38), we can find the expected reachable sets of $M_{1}$ and $M_{3}$ contain $\left[-a_{T, \max }, 0\right]$ and $\left[0, a_{T, \max }\right]$ respectively. Also, their initial reachable sets contain $\left[-a_{T, \max }, 0\right]$ and $\left[0, a_{T, \max }\right]$ respectively according to Eq. (9). Note that the random stepmaneuvering acceleration meets $a_{t}(t) \in\left[-a_{T, \max }, 0\right]$ or $a_{t}(t) \in\left[0, a_{T, \text { max }}\right]$, so the missile team is able to keep the target in the reachable set of $M_{1}$ or $M_{3}$ no matter when the target starts the step maneuver.

To further explain this, Fig. 10 presents the ZEM s of the three missiles against the step-maneuvering target with $\tau=3 s$ and $u=-1$, and Fig. 11 provides the reachable sets of missile $M_{1}$. From Fig. 10, we can see the ZEM s of $M_{1}$ and $M_{3}$ corresponding to the three guidance strategies all show the tendency of converging to 0 before the step maneuver stars, but they result from different reasons. For APN and DGL, it is because they assume the target will remain non-maneuvering. While for PCS, from the reachable set of $M_{1}$ shown in Fig. 11, we can see making the reachable set be as expected is also considered when trying to reduce the ZEM s. As a result, when the target starts step maneuver at $\tau=3$, $-a_{T, \max }$ has been outside of the reachable sets of APN and DGL, but still in the reachable set of $M_{1}$ adopting PCS, as shown in Fig. 11. Note that $M_{1}$ of PCS also fails to keep $-a_{T, \max }$ in its reachable set when it comes to the end of homing phase. It is mainly because in practice the guidance law shown in 
Eq. (25) can only be implemented discretely at the guidance period, and this will lead to inevitable guidance errors and bring the divergence of the reachable set when the time to go approaches zero.

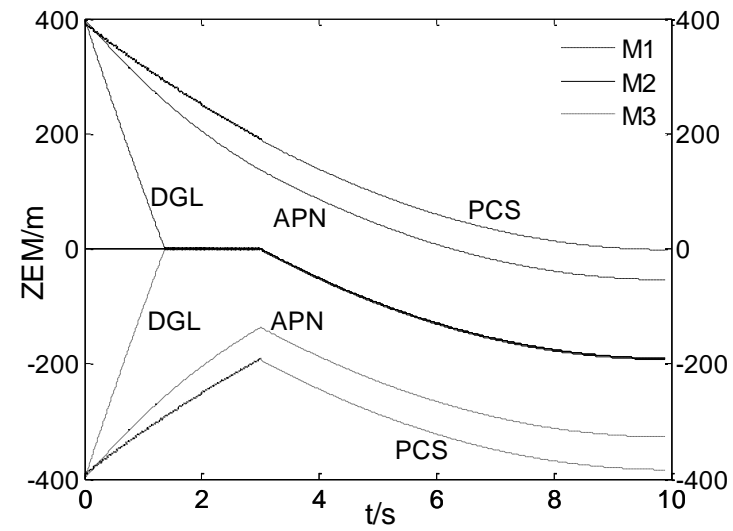

Fig. 10 ZEM against the random step maneuver: $u=-1$ and $\tau=3$

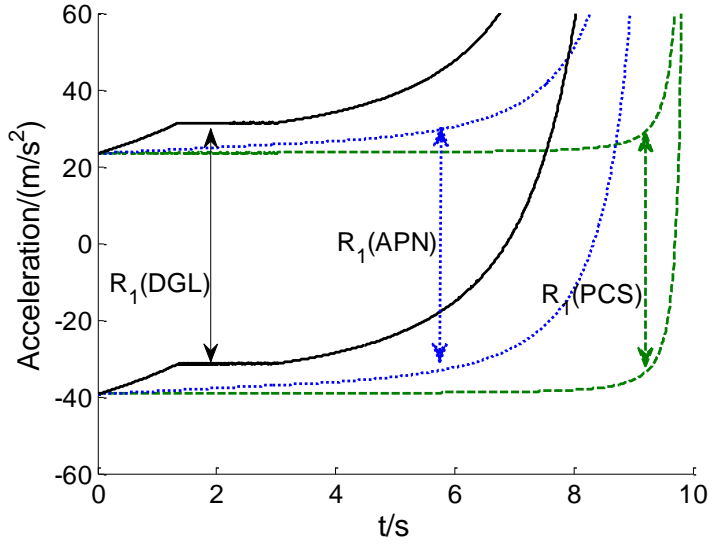

Fig. 11 The reachable sets of $M_{1}$ against the random step maneuver: $u=-1$ and $\tau=3$

The above simulation results indicate that PCS can guarantee the interceptions if the target executes constant maneuver or random step maneuver. However, if the target executes other forms of maneuver, such as bang-bang, the guidance performance of PCS will suffer degradation. The adaptive covering strategy (ACS) can effectively alleviate the degradation. Assuming the target executes bang-bang maneuver with single switch ${ }^{[20]}$, such as $u=-1,(0 \leq t<6 s)$ and $u=1,\left(6 s \leq t \leq t_{f}\right)$, Fig. 12 presents the ZEMs of the three missiles corresponding to PCS and ACS, and we can see only the missile $M_{2}$ in ACS reaches the acceptable miss distance at the end. Figs. 13 and 14 show the expected and current reachable sets of the three missiles in PCS and ACS. By comparison, we can see the first difference happens at around $t=3 s$, when $R_{2}^{*}$ is adjusted to be $\left[-3 a_{T, \max } / 5, a_{T, \max }\right]$ from $\left[-4 a_{T, \max } / 5,4 a_{T, \max } / 5\right]$ according to Eq. (27). Due to this adjustment, $a_{T, \max }$ is in the current and expected reachable sets of $M_{2}$ when the target changes its acceleration $a_{t}(t)=a_{T, \max },\left(6 \leq t \leq t_{f}\right)$. As a result, $M_{2}$ in ACS has a desirable miss distance. Besides, there are two adjustments near the end, which mainly result from the discrete guidance error. 


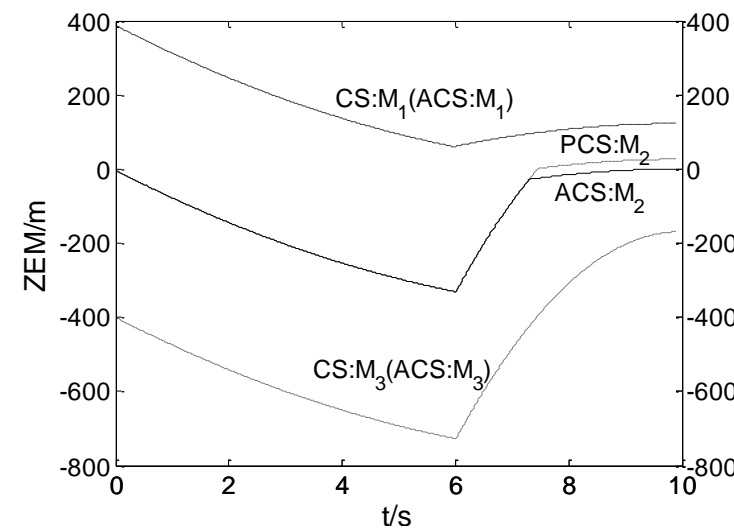

Fig. 12 ZEM of PCS and ACS against bangbang maneuver with single

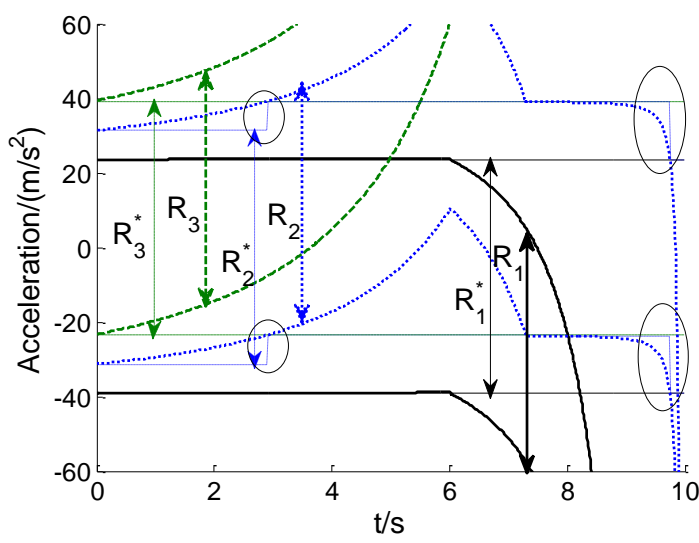

Fig. 14 Expected and current reachable sets of the three missiles in ACS

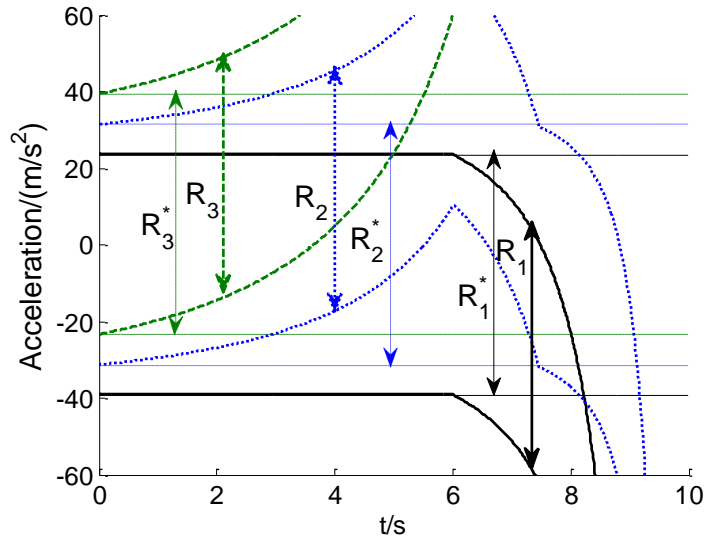

Fig. 13 Expected and current reachable sets of the three missiles in PCS

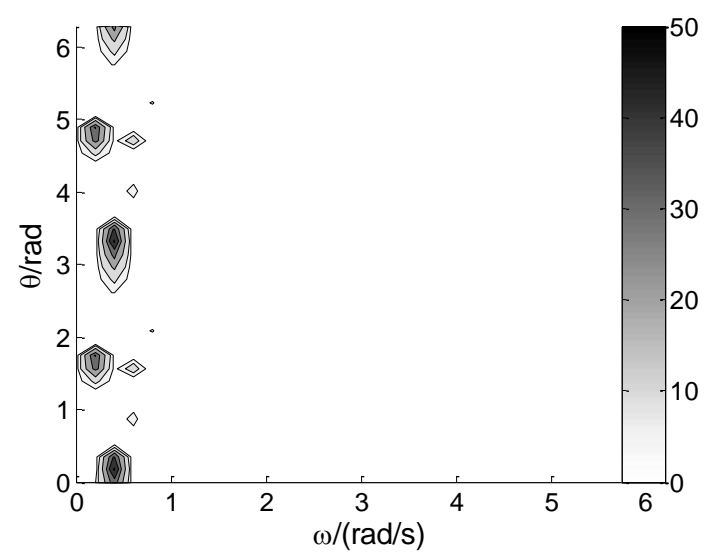

Fig. 15 Miss distance of ACS against bang-bang maneuver

To further verify the performance of the cooperative strategy, the bang-bang maneuver in more general form

$$
a_{t}=a_{t, \max } \operatorname{sign}(\sin (\omega t+\theta))
$$

is considered, where $\omega$ and $\theta$ indicates the angular frequency and initial phase of the maneuvering acceleration. Simulations with $\omega \in[0, \pi]$ and $\theta \in[0,2 \pi]$ are run, and Figs. 15, 16 and 17 present the miss distances of the missile team under the ACS, APN and DGL. It is obvious to find the guidance performance is jointly determined by $\omega$ and $\theta$, and generally the increase of $\omega$ is helpful to improve the performance of all the three guidance strategies. More importantly, we can find none of them can always guarantee the interception, but the missile team in ACS has smaller miss distance and higher capturing probability. Therefore, the performance of ACS is also evidently superior to that of APN and DGL when target executes random bang-bang maneuver. 


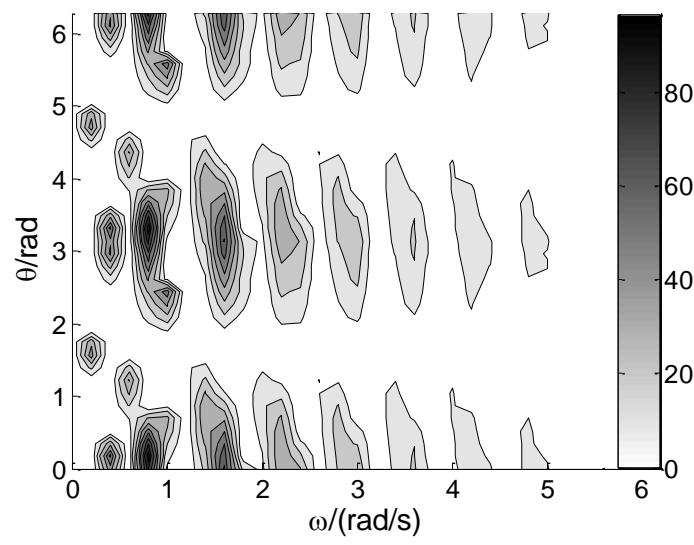

Fig. 16 Miss distance of APN against bang-bang maneuver

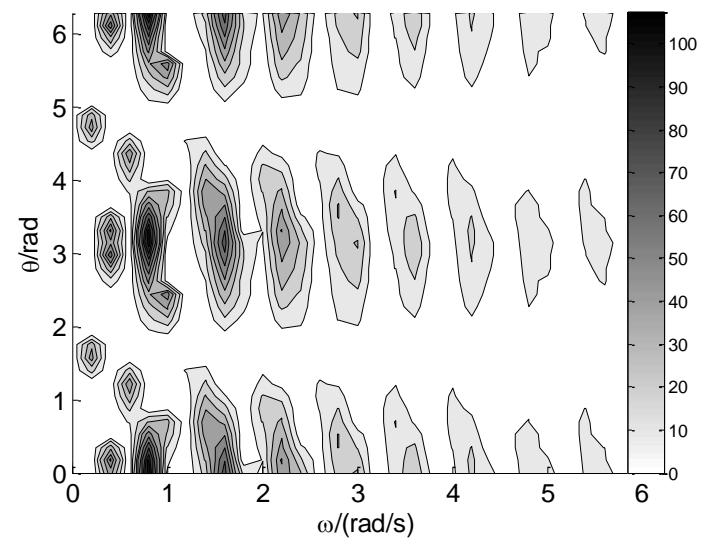

Fig. 17 Miss distance of DGL against bang-bang maneuver

Under the same target-maneuvering mode, Figs 18 and 19 show the influence of time delay, while Figs. 20 and 21 present the effect of missile number. Comparing the simulation results of Figs. 18 and 19 with that of Fig. 15, we find that increasing the time delay will aggravate the ACS performance. However, by comparing the results of Figs. 16, 17 with that of Fig. 19, the performance with time delay is still much better than that of the missile teams under APN and DGL, Besides, both Figs. 18 and 19 show the increase of target maneuvering frequency will decrease the negative effect of time delay. In Fig. 20, the two missiles are $M_{1}$ and $M_{3}$, while in Fig. 21 the four missiles consist of $M_{1}, M_{2}, M_{3}$ and the other one with initial position $[0,0]$ and velocity $[2,-0.04] \mathrm{km} / \mathrm{s}$. Comparing the results of Fig. 15 with that of Figs. 20 and 21, we can see that increasing the number of missiles is beneficial to performance improvement of ACS. However, we also find the number change from 3 to 4 does not bring as much performance improvement as that of the number change from 2 to 3 , which implies the improvement will become not obvious if the missile number is large enough.

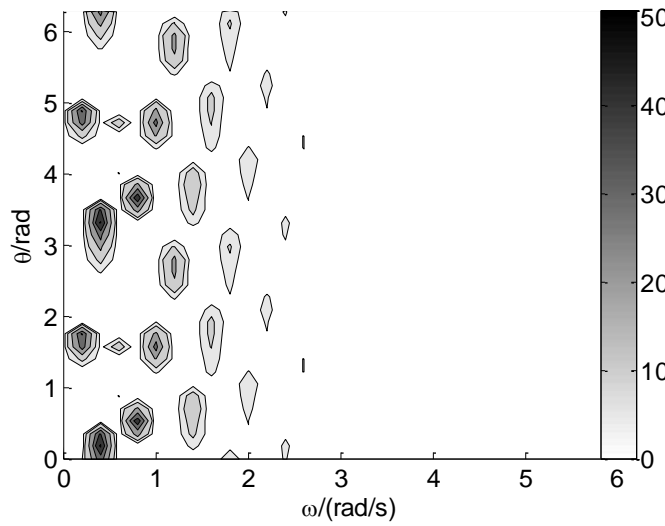

Fig. 18 Miss distance of ACS against bangbang maneuver with time delay $0.5 \mathrm{~s}$

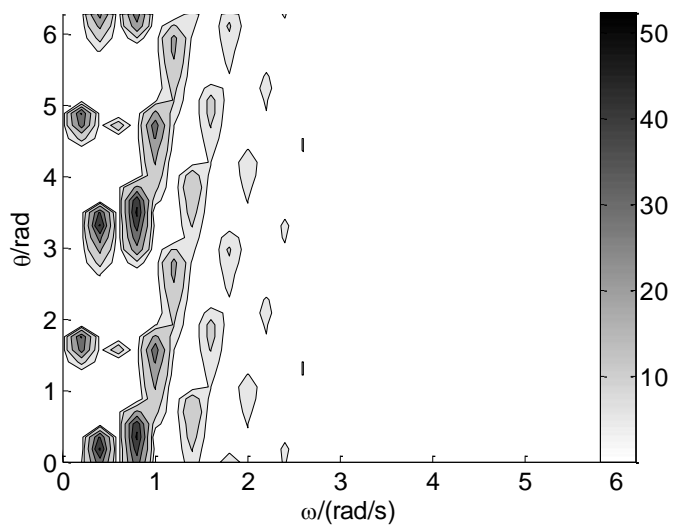

Fig. 19 Miss distance of ACS against bangbang maneuver with time delay $1 \mathrm{~s}$ 


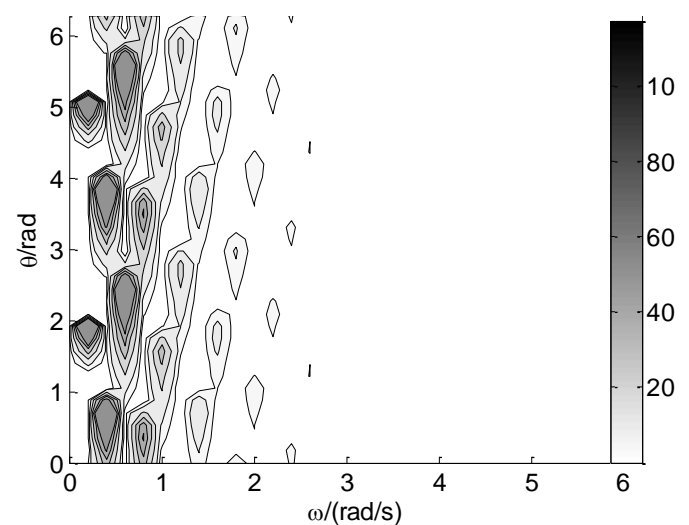

Fig. 20 Miss distance of ACS against bangbang maneuver with two missiles

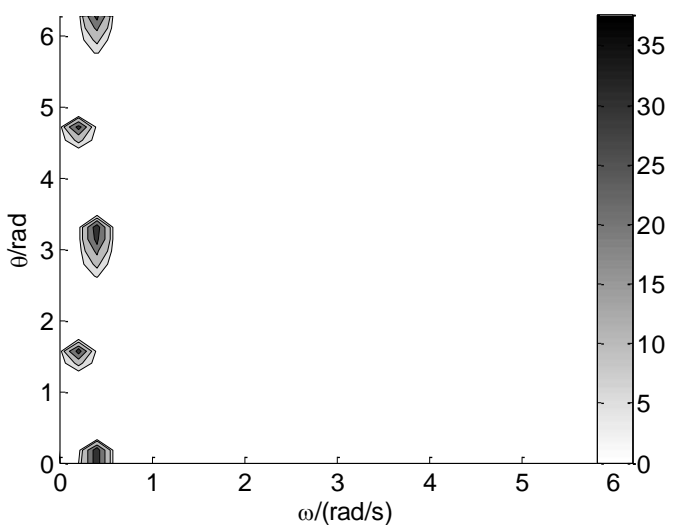

Fig. 21 Miss distance of ACS against bangbang maneuver with four missiles

The above simulation scenarios have verified the robust performance of ACS against time delay and different target maneuvering modes, but the performance against different initial engagement situations remains unknown. Therefore, the following scenario is constructed. It is assumed the initial states of $M_{1}, M_{2}$ and $M_{3}$ are the same as the above scenarios except for their lateral velocities, which are assumed to be uniformly distributed in the range $[-40,40] \mathrm{m} / \mathrm{s}$. As a result, their initial $Z E M$ $\mathrm{s}$ will be uniformly distributed in the range $[-400,400] \mathrm{m}$ approximately. Supposing the target executes bang-bang maneuver shown in Eq. (40), and both $\omega$ and $\theta$ are uniformly distributed in the range $[0,2 \pi]$. Under these assumptions, the simulation is run 1000 times, and the miss distances of the missile team under DGL, APN and ACS are obtained. Fig. 22 shows the corresponding probability distribution function of the miss distance. We can see the probability of reaching small miss distance for ACS is far larger than that of DGL and APN, which proves the robust performance of ACS again.

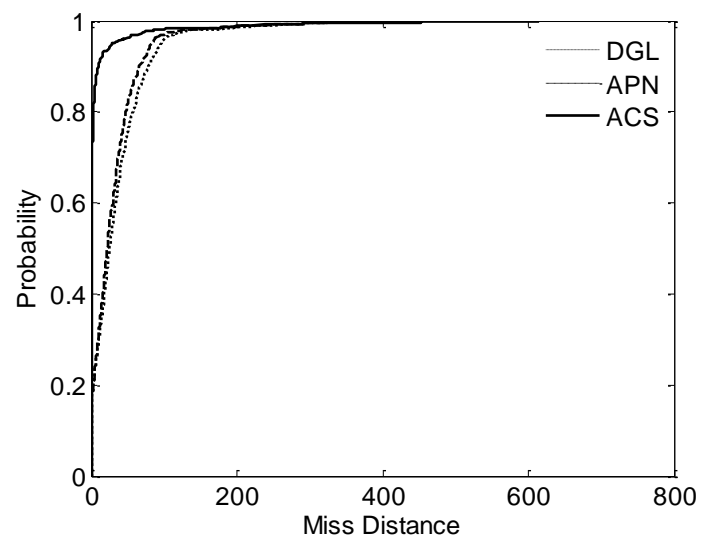

Fig. 22 Probability distribution function about miss distance

\section{Conclusion}

In this paper, a new cooperative guidance scheme is proposed for multiple missiles with inferior maneuverability to intercept the uncertain maneuvering target. The guidance goal is described as 
cooperatively covering the target maneuvering range with the joint reachable set of missile-team. Two different cooperative guidance strategies corresponding to the missile teams without and with communication capability are designed respectively, followed by the analysis of their inherent limitations. Simulations prove the validness of preprogrammed covering strategy against the constant maneuvering and the random step-maneuvering target, although the maximum maneuverability of each interception missile is inferior to that of the target. As for the other maneuvering modes, such as bang-bang maneuver, the adaptive covering strategy shows better adaptability than the preprogrammed covering strategy, and has the smaller miss distance and higher successful interception probability than that of APN and DGL.

\section{Conflict of interest statement}

The authors have no competing interests.

\section{Reference}

[1] Murtaugh, S. A., Criel, H. E. "Fundamentals of Proportional Navigation," IEEE Spectrum 3, No. 12,1966, pp. $75-85$.

[2] Guelman, M., “A Qualitative Study of Proportional Navigation,” IEEE Transactions on Aerospace and Electronic Systems AES-7, No. 4, 1971, pp. 637-43.

[3] Garber, V., "Optimal Intercept Laws for Accelerating Targets,” AIAA Journal, Vol. 6, No. 11, 1968, pp. 2196-2198.

[4] Zarchan, P., "Tactical and Strategic Missile Guidance (6th edition),” AIAA Inc., New York, 2012, Chaps. 3,8 .

[5] Cottrell, G. R., "Optimal Guidance for Short-Range Tactical Missiles,” AIAA Journal, Vol. 9, No. 6, 1971, pp. 1414-1415.

[6] Gutman, S., Leitmann, G., "Optimal strategies in the neighborhood of a collision course," AIAA Journal, Vol. 14, No.9, 1976, pp. 1210-1212.

[7] Shinar, J., Gutman. S., "Three-dimensional optimal pursuit and evasion with bounded control," IEEE Transactions on Automatic Control, Vo.25, No.3, 1980, pp. 492-496.

[8] Levant, A., "Principles of 2-Sliding Mode Design," Automatica, Vol.43, No. 4, 2007, pp. 576-86.

[9] Zhou, D., Sun, S., Teo, K. L., "Guidance Laws with Finite Time Convergence," Journal of Guidance, Control, and Dynamics, Vol.32, No. 6, 2009, pp. 1838-46 
[10] Yao, Y., Zheng, T. Y., He F. H., et al. "Several hot issues and challenges in terminal guidance of flight vehicles", Acta Aeronautica et Astronautica Sinica, Vol.36, No.8, 2015, pp. 2696-2716.

[11] Jeon, I. S., Lee, J. I, Tahk, M. J. “Impact-time-control guidance law for anti-ship missiles,” IEEE Transactions Control System Technology, Vol. 14, No. 2, 2006, pp. 260-266.

[12] Lee, J. I, Jeon, I. S, Tahk, M. J., “Guidance Law to Control Impact Time and Angle,” IEEE Transactions on Aerospace and Electronic Systems, Vol. 43, No. 1, 2007, pp. 301-310.

[13] Harl, N., Balakrishnan, S. N., "Impact Time and Angle Guidance With Sliding Mode Control," IEEE Transactions on Aerospace and Electronic Systems, Vol. 20, No. 6, 2012,pp. 1436-1449.

[14] Zhao, S., Zhou, R., “Cooperative Guidance for Multi-missile Salvo Attack,” Chinese Journal of Aeronautics, Vol. 21, No. 6, 2008, pp. 533-539.

[15] Jeon, I. S., Lee, J.I., “Homing Guidance Law for Cooperative Attack of Multiple Missiles,” Journal of Guidance, Control, and Dynamics, Vol. 33, No. 1,2010, pp. 275-280

[16] Zhao, S., Zhou, R., Wei, C., Ding, Q., "Design of Time-constrained Guidance Laws via Virtual Leader Approach,” Chinese Journal of Aeronautics, Vol. 23, No. 1, 2010. pp. 103-108.

[17] R. a. Best and J. P. Norton, "Predictive Missile Guidance," Journal of Guidance, Control, and Dynamics, Vol. 23, No.3, 2000, pp. 539-46.

[18] Dionne, D., Michalska, H., and Rabbath, C. A., "Predictive Guidance for Pursuit-Evasion Engagements Involving Multiple Decoys,” Journal of Guidance, Control and Dynamics, Vol. 30, No. 5, 2007, pp. 1277-1286.

[19] Zhai, C., He, F., Hong, Y., Wang, L., and Yao, Y., "Coverage-Based Interception Algorithm of Multiple Interceptors against the Target Involving Decoys", Journal of Guidance, Control, and Dynamics, Vol. 39, No. 7. 2016, pp. 1647-1653.

[20] Shaferman, V., Oshman, Y., "Stochastic Cooperative Interception Using Information Sharing Based on Engagement Staggering," Journal of Guidance, Control, and Dynamics, Vol. 39, No. 9 2016, pp. 2127-41.

[21] Wang. L, Y. Yu, He. F., Liu. K., “A novel cooperative mid-course guidance scheme for multiple intercepting missiles", Chinese Journal of Aeronautics, 2017.(Under publish)

[22] Richard V. Lawrence. "Interceptor Line-of-Sight Rate Steering: Necessary Conditions for a Direct Hit", Journal of Guidance, Control, and Dynamics, Vol. 21, No. 3 (1998), pp. 471-476.

[23] Shinar, J. and Shima, T., "Nonorthodox guidance law development approach for intercepting 
maneuvering targets,” Journal of Guidance, Control and Dynamics, Vol. 25, No. 4 Jul-Aug. 2002, pp. 658-666.

[24] Shima, T. and Shinar, J.. "Time-Varying Linear Pursuit-Evasion Game Models with Bounded Controls,” Journal of Guidance, Control, and Dynamics, Vol. 25, No. 3, May-Jun. 2002, pp. 425432.

[25] Shinar J., Turetsky V., and Oshman Y., "Integrated Estimation/Guidance Design Approach for Improved Homing Against Randomly Maneuvering Targets,” Journal of Guidance, Control, and Dynamics, Vol.30, No. 1, Jan-Feb. 2007, pp. 154-61. 
2018-07-02

\section{Cooperative interception strategy for multiple inferior missiles against one highly maneuvering target}

Su, Wenshan

Elsevier

Wenshan Su, Hyo-Sang Shin, Lei Chen and Antonios Tsourdos. Cooperative interception strategy for multiple inferior missiles against one highly maneuvering target. Aerospace Science and Technology, Volume 80, Issue September, 2018, Pages 91-100

https://doi.org/10.1016/j.ast.2018.06.026

Downloaded from Cranfield Library Services E-Repository 